\title{
Cooperative Interference Management with MISO Beamforming
}

\author{
Rui Zhang and Shuguang Cui
}

\begin{abstract}
This correspondence studies the downlink transmission in a multi-cell system, where multiple base stations (BSs) each with multiple antennas cooperatively design their respective transmit beamforming vectors to optimize the overall system performance. For simplicity, it is assumed that all mobile stations (MSs) are equipped with a single antenna each, and there is one active MS in each cell at one time. Accordingly, the system of interests can be modeled by a multiple-input single-output (MISO) interference channel (IC), termed as MISO-IC, with interference treated as noise. We propose a new method to characterize different rate-tuples for active MSs on the Pareto boundary of the achievable rate region for the MISO-IC, by exploring the relationship between the MISO-IC and the cognitive radio (CR) MISO channel. We show that each Pareto-boundary rate-tuple of the MISO-IC can be achieved in a decentralized manner when each of the BSs attains its own channel capacity subject to a certain set of interference-power constraints (also known as interference-temperature constraints in the CR system) at the other MS receivers. Furthermore, we show that this result leads to a new decentralized algorithm for implementing the multi-cell cooperative downlink beamforming.
\end{abstract}

\section{Index Terms}

Beamforming, cooperative multi-cell system, interference channel, multi-antenna, Pareto optimal, rate region.

\section{INTRODUCTION}

Conventional wireless mobile networks are designed with a cellular architecture, where base stations (BSs) from different cells control communications for their associated mobile stations (MSs) independently. The resulting intercell interference is treated as additive noise and minimized by applying a predesigned frequency reuse pattern such that the same frequency band is reused only by non-adjacent cells. Due to the rapidly growing demand for high-rate wireless multimedia applications, conventional cellular networks have been pushed towards their throughput limits. Consequently, many beyond-3G wireless technologies such as WiMAX and 3GPP UMTS Long Term Evolution (LTE) have relaxed the constraint on the frequency reuse such that the total frequency band becomes available for reuse by all cells. However, this factor-one frequency reuse pattern renders the overall network performance limited by the inter-cell interference; consequently, more sophisticated interference management techniques with multi-cell cooperation become

This work was presented in part at IEEE Wireless Communications and Networking Conference (WCNC), Sydney, Australia, April 18-21, 2010 .

R. Zhang is with the Institute for Infocomm Research, A*STAR, Singapore and the Department of Electrical and Computer Engineering, National University of Singapore, Singapore (e-mail: rzhang@i2r.a-star.edu.sg).

S. Cui is with the Department of Electrical and Computer Engineering, Texas A\&M University, Texas, USA (e-mail: cui@ece.tamu.edu). 
crucial. Among others, one effective method to cope with the inter-cell interference in the cellular network is via joint signal processing across different BSs. In this correspondence, we study a particular type of multi-BS cooperation for the downlink transmission, where we are interested in evaluating the benefit in terms of network throughput by cooperatively optimizing the transmit beamforming vectors for different BSs each with multiple antennas. Notice that the problem setup of our work is different from that for a fully cooperative multi-cell system considered in, e.g., [1]-[6], where a central processing unit is assumed with the global knowledge of all the required downlink channels and user messages to jointly design the transmitted signals for all BSs. In contrast, our work focuses on the decentralized implementation of the multi-cell cooperative downlink beamforming assuming only the local message and neighboring-channel knowledge at each BS, which is more practical than implementing the full baseband-level coordination. It is worth noting that decentralized multi-cell cooperative downlink beamforming has been studied in [7] to minimize the total power consumption of all BSs to meet with MSs' individual signal-to-interference-plus-noise ratio (SINR) targets, based on the uplink-downlink beamforming duality. In this work, we provide a different design approach for rate-optimal strategies in decentralized multi-cell cooperative beamforming.

For the purpose of exposition, in this work we consider a simplified scenario, where each MS is equipped with a single antenna, and at any given time there is only one active MS in each cell (over a particular frequency band). Accordingly, we can model the multi-cell cooperative downlink transmission system as a multiple-input single-output (MISO) Gaussian interference channel (IC), termed as MISO-IC. From an information-theoretic viewpoint, the capacity region of the Gaussian IC, which constitutes all the simultaneously achievable rates for all users, is still unknown in general [8], while significant progresses have recently been made on approaching this limit [9]. Capacity-approaching techniques for the Gaussian IC in general require certain signal-level encoding/decoding cooperations among the users, while a more pragmatic approach that leads to suboptimal achievable rates of the users is to allow only single-user encoding and decoding by treating the interference from other users as additive Gaussian noise. In this work, we adopt the latter approach to study the design of cooperative transmit beamforming for the MISO-IC. Particularly, we focus on the design criterion to achieve different rate-tuples for the users on the Pareto boundary of the achievable rate region for the MISO-IC. Due to the coupled signal structure, the achievable rate region for the MISO-IC with interference treated as noise is in general a non-convex set. 1 which renders the joint optimization of beamforming vectors to achieve different Pareto-boundary rate-tuples a challenging task. Note that this problem has been studied in [10], where for the special two-user case, it was shown that the optimal transmit beamforming vector to achieve a Pareto-boundary rate-pair for the MISO-IC can be expressed as a linear combination of the zero-forcing (ZF) and

\footnotetext{
${ }^{1}$ It is noted that the non-convex rate region is obtained without time-sharing (convex-hull operation) between different achievable rate-tuples. With time-sharing, the achievable rate region will become a convex set.
} 
maximum-ratio transmission (MRT) beamformers. The rate maximization for the IC with interference treated as noise has also been studied in the literature via various "pricing" algorithms (see, e.g., [11] and references therein), while in general the price-based approach does not achieve the Pareto-optimal rates for the MISO-IC. In [12], the maximum sum-rate for the Gaussian IC with interference treated as noise is characterized in terms of degrees of freedom (DoF) over the interference-limited regime.

In this correspondence, we develop a new parametrical characterization of the Pareto boundary for the MISO-IC in terms of the interference-power levels at all receivers caused by different transmitters, also known as the interference temperature (IT) levels in the newly emerging "cognitive radio (CR)" type of applications [13]. We show that each Pareto-boundary rate-tuple can be achieved in a decentralized manner when each of the users maximizes its own MISO channel capacity subject to a certain set of IT constraints at the other users' receivers, which is identical to the CR MISO channel transmit optimization problem studied in [14] and thus shares the same solution structure. We derive new closed-form solutions for the optimal transmit covariance matrices of all users to achieve an arbitrary rate-tuple on the Pareto boundary of the MISO-IC rate region, from which we see that the optimal transmit covariance matrices should all be rank-one (i.e., beamforming is optimal) 2 Furthermore, we derive the conditions that are necessary for any particular set of mutual IT constraints across all users to guarantee a Pareto-optimal rate-tuple for the MISO-IC. Based on these conditions, we propose a new decentralized algorithm for implementing the multi-cell cooperative downlink beamforming. For this algorithm, all different pairs of BSs independently search for their mutually desirable IT constraints (with those for the MSs associated with the other BSs fixed), under which their respective beamforming vectors are optimized to maximize the individual transmit rates. This algorithm improves the rates for the BSs in a pairwise manner until the transmit rates for all BSs converge with their mutual IT levels.

Notation: $\boldsymbol{I}$ and $\mathbf{0}$ denote the identity matrix and the all-zero matrix, respectively, with appropriate dimensions. For a square matrix $\boldsymbol{S}, \operatorname{Tr}(\boldsymbol{S}),|\boldsymbol{S}|, \boldsymbol{S}^{-1}$, and $\boldsymbol{S}^{1 / 2}$ denote the trace, determinant, inverse, and square-root of $\boldsymbol{S}$, respectively; and $\boldsymbol{S} \succeq 0$ means that $S$ is positive semi-definite [16]. $\operatorname{Diag}(\boldsymbol{a})$ denotes a diagonal matrix with the diagonal elements given by $\boldsymbol{a}$. For a matrix $\boldsymbol{M}$ of arbitrary size, $\boldsymbol{M}^{H}, \boldsymbol{M}^{T}$, and $\operatorname{Rank}(\boldsymbol{M})$ denote the Hermitian transpose, transpose, and rank of $M$, respectively. $\mathbb{E}[\cdot]$ denotes the statistical expectation. The distribution of a circularly symmetric complex Gaussian (CSCG) random vector with the mean vector $\boldsymbol{x}$ and the covariance matrix $\boldsymbol{\Sigma}$ is denoted by $\mathcal{C N}(\boldsymbol{x}, \boldsymbol{\Sigma})$; and $\sim$ stands for "distributed as". $\mathbb{C}^{m \times n}$ denotes the space of $m \times n$ complex matrices. $\|\boldsymbol{x}\|$ denotes the Euclidean norm of a complex vector (scalar) $\boldsymbol{x}$. The $\log (\cdot)$ function is with base 2 by default.

\footnotetext{
${ }^{2}$ We thank the anonymous reviewer who brought our attention to [15], in which the authors also showed the optimality of beamforming to achieve the Pareto-boundary rates for the Gaussian MISO-IC with interference treated as noise, via a different proof technique.
} 


\section{System Model}

We consider the downlink transmission in a cellular network consisting of $K$ cells, each having a multi-antenna BS to transmit independent messages to one active single-antenna MS. It is assumed that all BSs share the same narrow-band spectrum for downlink transmission. Accordingly, the system under consideration can be modeled by a $K$-user MISO-IC. It is assumed that the BS in the $k$ th cell, $k=1, \ldots, K$, is equipped with $M_{k}$ transmitting antennas, $M_{k} \geq 1$. The discrete-time baseband signal received by the active MS in the $k$ th cell is then given by

$$
y_{k}=\boldsymbol{h}_{k k}^{H} \boldsymbol{x}_{k}+\sum_{j \neq k}^{K} \boldsymbol{h}_{j k}^{H} \boldsymbol{x}_{j}+z_{k}
$$

where $\boldsymbol{x}_{k} \in \mathbb{C}^{M_{k} \times 1}$ denotes the transmitted signal from the $k$ th BS; $\boldsymbol{h}_{k k}^{H} \in \mathbb{C}^{1 \times M_{k}}$ denotes the direct-link channel for the $k$ th MS, while $\boldsymbol{h}_{j k}^{H} \in \mathbb{C}^{1 \times M_{j}}$ denotes the cross-link channel from the $j$ th BS to the $k$ th MS, $j \neq k$; and $z_{k}$ denotes the receiver noise. It is assumed that $z_{k} \sim \mathcal{C N}\left(0, \sigma_{k}^{2}\right), \forall k$, and all $z_{k}$ 's are independent.

We assume independent encoding across different BSs and thus $\boldsymbol{x}_{k}$ 's are independent over $k$. It is further assumed that a Gaussian codebook is used at each BS and $\boldsymbol{x}_{k} \sim \mathcal{C N}\left(\mathbf{0}, \boldsymbol{S}_{k}\right), k=1, \ldots, K$, where $\boldsymbol{S}_{k}=\mathbb{E}\left[\boldsymbol{x}_{k} \boldsymbol{x}_{k}^{H}\right]$ denotes the transmit covariance matrix for the $k$ th BS, with $\boldsymbol{S}_{k} \in \mathbb{C}^{M_{k} \times M_{k}}$ and $\boldsymbol{S}_{k} \succeq 0$. Notice that the CSCG distribution has been assumed for all the transmitted signals 3 Furthermore, the interferences at all the receivers caused by different transmitters are treated as Gaussian noises. Thus, for a given set of transmit covariance matrices of all BSs, $\boldsymbol{S}_{1}, \ldots, \boldsymbol{S}_{K}$, the achievable rate of the $k$ th MS is expressed as

$$
R_{k}\left(\boldsymbol{S}_{1}, \ldots, \boldsymbol{S}_{K}\right)=\log \left(1+\frac{\boldsymbol{h}_{k k}^{H} \boldsymbol{S}_{k} \boldsymbol{h}_{k k}}{\sum_{j \neq k} \boldsymbol{h}_{j k}^{H} \boldsymbol{S}_{j} \boldsymbol{h}_{j k}+\sigma_{k}^{2}}\right) .
$$

Next, we define the achievable rate region for the MISO-IC to be the set of rate-tuples for all MSs that can be simultaneously achievable under a given set of transmit-power constraints for the BSs, denoted by $P_{1}, \ldots, P_{K}$ :

$$
\mathcal{R} \triangleq \bigcup_{\left\{\boldsymbol{S}_{k}\right\}: \operatorname{Tr}\left(\boldsymbol{S}_{k}\right) \leq P_{k}, k=1, \ldots, K}\left\{\left(r_{1}, \ldots, r_{K}\right): 0 \leq r_{k} \leq R_{k}\left(\boldsymbol{S}_{1}, \ldots, \boldsymbol{S}_{K}\right), k=1, \ldots, K\right\} .
$$

The upper-right boundary of this region is called the Pareto boundary, since it consists of rate-tuples at which it is impossible to improve a particular user's rate, without simultaneously decreasing the rate of at least one of the other users. More precisely, the Pareto optimality of a rate-tuple is defined as follows [10].

Definition 2.1: A rate-tuple $\left(r_{1}, \ldots, r_{K}\right)$ is Pareto optimal if there is no other rate-tuple $\left(r_{1}^{\prime}, \ldots, r_{K}^{\prime}\right)$ with $\left(r_{1}^{\prime}, \ldots, r_{K}^{\prime}\right) \geq$ $\left(r_{1}, \ldots, r_{K}\right)$ and $\left(r_{1}^{\prime}, \ldots, r_{K}^{\prime}\right) \neq\left(r_{1}, \ldots, r_{K}\right)$ (the inequality is component-wise).

In this work, we consider the scenario where multiple BSs in the cellular network cooperatively design their transmit covariance matrices in order to minimize the effect of the inter-cell interference on the overall network throughput. In

${ }^{3}$ It is worth noting that in [17] the authors point out that the CSCG distribution for the transmitted signals is in general non-optimal for the Gaussian IC with interference treated as noise, since it can be shown that the complex Gaussian but not circularly symmetric distribution can achieve larger rates than the symmetric distribution for some particular channel realizations. 


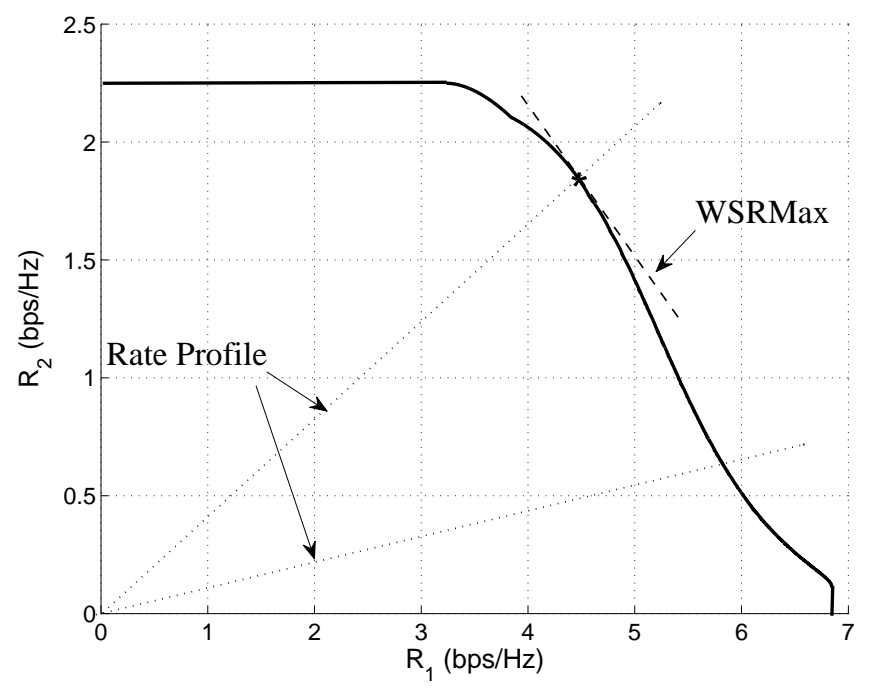

Fig. 1. Achievable rate region and Pareto boundary for a two-user MISO Gaussian IC with interference treated as noise.

particular, we are interested in the design criterion to achieve different Pareto-optimal rate-tuples for the corresponding MISO-IC defined as above.

It is worth noting that in prior works on characterizing the Pareto boundary for the MISO-IC with interference treated as noise (see, e.g., [10] and references therein), it has been assumed (without proof) that the optimal transmit strategy for users to achieve any rate-tuple on the Pareto boundary is beamforming, i.e., $\boldsymbol{S}_{k}$ is a rank-one matrix for all $k$ 's. Under this assumption, we can express $\boldsymbol{S}_{k}$ as $\boldsymbol{S}_{k}=\boldsymbol{w}_{k} \boldsymbol{w}_{k}^{H}, k=1, \ldots, K$, where $\boldsymbol{w}_{k} \in \mathbb{C}^{M_{k} \times 1}$ denotes the beamforming vector for the $k$ th user. Similarly as in the general case with $\operatorname{Rank}\left(\boldsymbol{S}_{k}\right) \geq 1$, the achievable rates and rate region of the MISO-IC with transmit beamforming (BF) can be defined in terms of $\boldsymbol{w}_{k}$ 's. However, it is not evident whether the BF case bears the same Pareto boundary as the general case with $\operatorname{Rank}\left(\boldsymbol{S}_{k}\right) \geq 1$ for the MISO-IC. In this work, we will show that this is indeed the case (see Section $\amalg I I)$. Accordingly, we can choose to use the rate and rate-region expressions in terms of either $\boldsymbol{S}_{k}$ 's or $\boldsymbol{w}_{k}$ 's to characterize the Pareto boundary of the MISO-IC, for the rest of this correspondence.

In the following, we review some existing approaches to characterize the Pareto boundary for the MISO-IC with interference treated as noise. For the purpose of illustration, in Fig. 1, we show the achievable rate region for a two-user MISO Gaussian IC with interference treated as noise (prior to any time-sharing of achievable rate-pairs), which is observed to be non-convex. A commonly adopted method to obtain the Pareto boundary for the MISO-IC is via solving a sequence of weighted sum-rate maximization (WSRMax) problems, each for a given set of user rate 
weights, $\mu_{k} \geq 0, \forall k$, and given by

$$
\begin{aligned}
\operatorname{Max}_{\left\{\boldsymbol{w}_{k}\right\}} & \sum_{k=1}^{K} \mu_{k} \log \left(1+\gamma_{k}\left(\boldsymbol{w}_{1}, \ldots, \boldsymbol{w}_{K}\right)\right) \\
\text { s.t. } & \left\|\boldsymbol{w}_{k}\right\|^{2} \leq P_{k}, k=1, \ldots, K
\end{aligned}
$$

where $\gamma_{k}\left(\boldsymbol{w}_{1}, \ldots, \boldsymbol{w}_{K}\right)$ is the receiver SINR for the $k$ th user defined as

$$
\gamma_{k}\left(\boldsymbol{w}_{1}, \ldots, \boldsymbol{w}_{K}\right)=\frac{\left\|\boldsymbol{h}_{k k}^{H} \boldsymbol{w}_{k}\right\|^{2}}{\sum_{j \neq k}\left\|\boldsymbol{h}_{j k}^{H} \boldsymbol{w}_{j}\right\|^{2}+\sigma_{k}^{2}}, k=1, \ldots, K .
$$

It is easy to verify that this problem is non-convex, and thus cannot be solved efficiently. In addition, the above method cannot guarantee the finding of all Pareto-boundary points for the MISO-IC (cf. Fig. 11).

An alternative method to characterize the complete Pareto boundary for the MISO-IC is based on the concept of rate profile [18]. Specifically, any rate-tuple on the Pareto boundary of the rate region can be obtained via solving the following optimization problem with a particular rate-profile vector, $\boldsymbol{\alpha}=\left(\alpha_{1}, \ldots, \alpha_{K}\right)$ :

$$
\begin{aligned}
\underset{R_{\text {sum }},\left\{\boldsymbol{w}_{k}\right\}}{\operatorname{Max}} & R_{\text {sum }} \\
\text { s.t. } & \log \left(1+\gamma_{k}\left(\boldsymbol{w}_{1}, \ldots, \boldsymbol{w}_{K}\right)\right) \geq \alpha_{k} R_{\text {sum }}, \quad k=1, \ldots, K \\
& \left\|\boldsymbol{w}_{k}\right\|^{2} \leq P_{k}, \quad k=1, \ldots, K
\end{aligned}
$$

with $\alpha_{k}$ denoting the target ratio between the $k$ th user's achievable rate and the users' sum-rate, $R_{\text {sum }}$. Without loss of generality, we assume that $\alpha_{k} \geq 0, \forall k$, and $\sum_{k=1}^{K} \alpha_{k}=1$. For a given $\boldsymbol{\alpha}$, let the optimal solution of Problem (6) be denoted by $R_{\mathrm{sum}}^{*}$. Then, it follows that $R_{\mathrm{sum}}^{*} \cdot \boldsymbol{\alpha}$ must be the corresponding Pareto-optimal rate-tuple, which can be geometrically viewed as (cf. Fig. 1) the intersection between a ray in the direction of $\boldsymbol{\alpha}$ and the Pareto boundary of the rate region. Thereby, with different $\boldsymbol{\alpha}$ 's, solving Problem (6) yields the complete Pareto boundary for the rate region, which does not need to be convex.

Next, we show that Problem (6) can be solved via solving a sequence of feasibility problems each for a fixed $r_{\text {sum }}$ and expressed as

$$
\begin{array}{ll}
\text { Find } & \left\{\boldsymbol{w}_{k}\right\} \\
\text { s.t. } & \log \left(1+\gamma_{k}\left(\boldsymbol{w}_{1}, \ldots, \boldsymbol{w}_{K}\right)\right) \geq \alpha_{k} r_{\mathrm{sum}}, \quad k=1, \ldots, K \\
& \left\|\boldsymbol{w}_{k}\right\|^{2} \leq P_{k}, \quad k=1, \ldots, K .
\end{array}
$$

If the above problem is feasible for a given sum-rate target, $r_{\mathrm{sum}}$, it follows that $R_{\mathrm{sum}}^{*} \geq r_{\mathrm{sum}}$; otherwise, $R_{\mathrm{sum}}^{*}<r_{\mathrm{sum}}$. Thus, by solving Problem (7) with different $r_{\text {sum }}$ 's and applying the simple bisection method [16] over $r_{\text {sum }}, R_{\text {sum }}^{*}$ can be obtained for Problem (6). Let $\bar{\gamma}_{k}=2^{\alpha_{k} r_{\mathrm{sum}}}-1, k=1, \ldots K$. Then, for Problem (7), we can replace the rate 
constraints by the equivalent SINR constraints given by

$$
\gamma_{k}\left(\boldsymbol{w}_{1}, \ldots, \boldsymbol{w}_{K}\right) \geq \bar{\gamma}_{k}, \quad k=1, \ldots, K
$$

Similarly as shown in [19], the resultant feasibility problem can be transformed into a second-order cone programming (SOCP) problem, which is convex and can be solved efficiently [20].

\section{Characterizing Pareto Boundary for MiSO-IC via Interference Temperature Control}

In this section, instead of investigating centralized approaches, we present a new method to characterize the Pareto boundary for the MISO-IC in a distributed fashion, by exploring its relationship with the CR MISO channel [14]. We start with the general-rank transmit covariance matrices $\boldsymbol{S}_{k}$ 's for the MISO-IC. First, we introduce a set of auxiliary variables, $\Gamma_{k j}, k=1, \ldots, K, j=1, \ldots, K, j \neq k$, where $\Gamma_{k j}$ is called the interference-power or interferencetemperature (IT) constraint from the $k$ th $\mathrm{BS}$ to $j$ th $\mathrm{MS}, j \neq k, \Gamma_{k j} \geq 0$. For notational convenience, let $\boldsymbol{\Gamma}$ be the vector consisting of all $K(K-1)$ different $\Gamma_{k j}$ 's, and $\boldsymbol{\Gamma}_{k}$ be the vector consisting of all $2(K-1)$ different $\Gamma_{k j}$ 's and $\Gamma_{j k}$ 's, $j=1, \ldots, K, j \neq k$, for any given $k \in\{1, \ldots, K\}$.

Next, we consider a set of parallel transmit covariance optimization problems, each for one of the $K$ BSs in the MISO-IC expressed as

$$
\begin{array}{cl}
\operatorname{Max}_{\boldsymbol{S}_{k}} & \log \left(1+\frac{\boldsymbol{h}_{k k}^{H} \boldsymbol{S}_{k} \boldsymbol{h}_{k k}}{\sum_{j \neq k} \Gamma_{j k}+\sigma_{k}^{2}}\right) \\
\text { s.t. } & \boldsymbol{h}_{k j}^{H} \boldsymbol{S}_{k} \boldsymbol{h}_{k j} \leq \Gamma_{k j}, \forall j \neq k \\
& \operatorname{Tr}\left(\boldsymbol{S}_{k}\right) \leq P_{k}, \quad \boldsymbol{S}_{k} \succeq 0
\end{array}
$$

where $k \in\{1, \ldots, K\}$. Note that in the above problem for a given $k, \boldsymbol{\Gamma}_{k}$ is fixed. For notational convenience, we denote the optimal value of this problem as $C_{k}\left(\boldsymbol{\Gamma}_{k}\right)$. If in the objective function of (9) we set $\Gamma_{j k}=\boldsymbol{h}_{j k}^{H} \boldsymbol{S}_{j} \boldsymbol{h}_{j k}, \forall j \neq k$, $C_{k}\left(\boldsymbol{\Gamma}_{k}\right)$ becomes equal to the maximum achievable rate of an equivalent MISO CR channel [14], where the $k$ th BS is the so-called "secondary" user transmitter, and all the other $K-1 \mathrm{BSs}$, indexed by $j=1, \ldots, K, j \neq k$, are the "primary" user transmitters, each of which has a transmit covariance matrix, $\boldsymbol{S}_{j}$, and its intended "primary" user

receiver is protected by the secondary user via the IT constraint: $\boldsymbol{h}_{k j}^{H} \boldsymbol{S}_{k} \boldsymbol{h}_{k j} \leq \Gamma_{k j}$. In [14], it was proved that the solution for Problem (9) is rank-one, i.e., beamforming is optimal, and in the special case of $K=2$ (i.e., one single primary user), a closed-form solution for the optimal beamforming vector was derived. In the following proposition, we provide a new closed-form solution for Problem (9) with arbitrary values of $K$, from which we can easily infer that beamforming is indeed optimal. 
Proposition 3.1: The optimal solution of Problem (9) is rank-one, i.e., $\boldsymbol{S}_{k}=\boldsymbol{w}_{k} \boldsymbol{w}_{k}^{H}$, and

$$
\boldsymbol{w}_{k}=\left(\sum_{j \neq k} \lambda_{k j} \boldsymbol{h}_{k j} \boldsymbol{h}_{k j}^{H}+\lambda_{k k} \boldsymbol{I}\right)^{-1} \boldsymbol{h}_{k k} \sqrt{p_{k}}
$$

where $\lambda_{k j}, j \neq k$, and $\lambda_{k k}$, are non-negative constants (dual variables) corresponding to the $k$ th BS's IT constraint for the $j$ th MS and its own transmit-power constraint, respectively, which are obtained as the optimal solutions for the dual variables in the dual problem of Problem (9); and $p_{k}$ is given by

$$
p_{k}=\left(\frac{1}{\ln 2}-\frac{\sum_{j \neq k} \Gamma_{j k}+\sigma_{k}^{2}}{\left\|\boldsymbol{A}_{k} \boldsymbol{h}_{k k}\right\|^{2}}\right)^{+} \frac{1}{\left\|\boldsymbol{A}_{k} \boldsymbol{h}_{k k}\right\|^{2}}
$$

where $\boldsymbol{A}_{k} \triangleq\left(\sum_{j \neq k} \lambda_{k j} \boldsymbol{h}_{k j} \boldsymbol{h}_{k j}^{H}+\lambda_{k k} \boldsymbol{I}\right)^{-1 / 2}$ and $(x)^{+} \triangleq \max (0, x)$.

Proof: Please see Appendix A

Now, we are ready to present a parametrical characterization of the Pareto boundary for the MISO-IC in terms of $\Gamma$ as follows.

Proposition 3.2: For any rate-tuple $\left(R_{1}, \ldots, R_{K}\right)$ on the Pareto boundary of the MISO-IC rate region defined in (3), which is achievable with a set of transmit covariance matrices, $\boldsymbol{S}_{1}, \ldots, \boldsymbol{S}_{K}$, there is a corresponding interferencepower constraint vector, $\boldsymbol{\Gamma} \geq 0$, with $\Gamma_{k j}=\boldsymbol{h}_{k j}^{H} \boldsymbol{S}_{k} \boldsymbol{h}_{k j}, \forall j \neq k, j \in\{1, \ldots, K\}$, and $k \in\{1, \ldots, K\}$, such that $R_{k}=C_{k}\left(\boldsymbol{\Gamma}_{k}\right), \forall k$, and $\boldsymbol{S}_{k}$ is the optimal solution of Problem (9) for the given $k$.

Proof: Please see Appendix B

From Proposition 3.2, it follows that the Pareto boundary for the MISO-IC is parameterized in terms of a lowerdimensional manifold over the non-negative real vector $\Gamma$, in comparison with that over the complex transmit covariance matrices, $\boldsymbol{S}_{k}$ 's, or with that over the complex beamforming vectors, $\boldsymbol{w}_{k}$ 's. Furthermore, by combining Propositions 3.1 and 3.2, it follows that beamforming is indeed optimal to achieve any rate-tuple on the Pareto boundary for the MISO-IC.

Remark 1: It is worth noting that the dimensionality reduction approach proposed in this work for characterizing the Pareto boundary of the MISO-IC is in spirit similar to that proposed in [10], where it has been shown that the transmit beamforming vectors to achieve any Pareto-boundary rate-tuple of the $K$-user MISO-IC with interference treated as noise can be expressed in the following forms:

$$
\boldsymbol{w}_{k}=\sum_{j=1}^{K} \xi_{k j} \boldsymbol{h}_{k j}, \quad k=1, \ldots, K
$$

where $\xi_{k j}$ 's are complex coefficients. Note that under the assumption of independent $\boldsymbol{h}_{k j}$ 's, the above beamforming structure is non-trivial only when $M_{k}>K$. For this case, from Remark 2 in Appendix A it is known that for the optimal beamforming structure given in (10), we have $\lambda_{k k}>0$. With this and by applying the matrix inversion lemma 
[21], it can be shown (the detailed proof is omitted here for brevity) that the optimal beamforming structure given by (10) is indeed in accordance with that given by (12). The main difference for these two methods to characterize the Pareto boundary for the MISO-IC lies in their adopted parameters: The method in our work uses $K(K-1)$ real $\Gamma_{k j}$ 's, while that in [10] uses $K(K-1)$ complex $\xi_{k j}$ 's. Note that $\Gamma_{k j}$ corresponds to the IT constraint from the $k$ th user transmitter to the $j$ th user receiver, whereas there is no practical meaning associated with $\xi_{k j}$. Consequently, as will be shown next, the proposed method in our work leads to a practical algorithm to implement the multi-cell cooperative downlink beamforming, via iteratively searching for mutually desirable IT constraints between different pairs of BSs.

\section{Decentralized Algorithm for Multi-Cell Cooperative Beamforming}

In this section, we develop a new decentralized algorithm that practically implements the multi-cell cooperative downlink beamforming based on the results derived in the previous section. It is assumed that each BS in the cellular network has the perfect knowledge of the channels from it to all MSs. Furthermore, it is assumed that all BSs operate according to the same protocol described as follows. Initially, a set of prescribed IT constraints in $\Gamma$ are set over the whole network, and the $k$ th BS is informed of its corresponding $\boldsymbol{\Gamma}_{k}, k=1, \ldots, K$. Accordingly, each BS sets its own transmit beamforming vector via solving Problem (9) and sets its transmit rate equal to the optimal objective value of Problem (9), which is achievable for its MS since the actual IT levels from the other BSs must be below their prescribed constraints. Then, by assuming that there is an error-free link between each pair of BSs, all different pairs of BSs start to communicate with each other for updating their mutual IT constraints (the details are given later in this section), under which each pair of BSs reset their respective beamforming vectors via solving Problem (9) such that the achievable rates for their MSs both get improved. Notice that each pair of updating BSs keeps the IT constraints for the MSs associated with the other BSs excluding this pair fixed; and as a result, the transmit rates for all the other MSs are not affected. Therefore, the above algorithm can be implemented in a pairwise decentralized manner across the BSs, while it converges when there are no incentives for all different pairs of BSs to further update their mutual IT constraints.

Next, we focus on the key issue on how to update the mutual IT constraints for each particular pair of BSs to guarantee the rate increase for both of their MSs. To resolve this problem, in the following proposition, we first provide the necessary conditions for any given $\Gamma \geq 0$ (component-wise) to correspond to a Pareto-optimal rate-tuple for the MISO-IC, which will lead to a simple rule for updating the mutual IT constraints between different pairs of BSs. Note that from Proposition 3.2, it follows that for any Pareto-optimal rate-tuple of the MISO-IC, there must exist a $\Gamma$ such that the optimal solutions of the problems given in 9 for all $k$ 's are the same as those for the general 
formulation of MISO-IC to achieve this rate-tuple. However, for any given $\Gamma \geq 0$, it remains unknown whether this value of $\boldsymbol{\Gamma}$ will correspond to a Pareto-optimal rate-tuple for the MISO-IC.

Proposition 4.1: For an arbitrarily chosen $\Gamma \geq 0$, if the optimal rate values of the problems in (9) for all $k$ 's, $C_{k}\left(\boldsymbol{\Gamma}_{k}\right)$ 's, are Pareto-optimal on the boundary of the MISO-IC rate region defined in (3), then for any pair of $(i, j), i \in$ $\{1, \ldots, K\}, j \in\{1, \ldots, K\}$, and $i \neq j$, it must hold that $\left|\boldsymbol{D}_{i j}\right|=0$, where $\boldsymbol{D}_{i j}$ is defined as

$$
\boldsymbol{D}_{i j}=\left[\begin{array}{cc}
\frac{\partial C_{i}\left(\boldsymbol{\Gamma}_{i}\right)}{\partial \Gamma_{i j}} & \frac{\partial C_{i}\left(\boldsymbol{\Gamma}_{i}\right)}{\partial \Gamma_{j i}} \\
\frac{\partial C_{j}\left(\boldsymbol{\Gamma}_{j}\right)}{\partial \Gamma_{i j}} & \frac{\partial C_{j}\left(\boldsymbol{\Gamma}_{j}\right)}{\partial \Gamma_{j i}}
\end{array}\right] .
$$

Proof: Please see Appendix [C]

Note that $\boldsymbol{D}_{i j}$ 's for all different pairs of $(i, j)$ can be obtained from the (primal and dual) solutions of the problems given in (9) for all k's with the given $\Gamma$ (for the details, please refer to Appendix (A). More specifically, we have

$$
\frac{\partial C_{i}\left(\boldsymbol{\Gamma}_{i}\right)}{\partial \Gamma_{i j}}=\lambda_{i j}
$$

where $\lambda_{i j}$ is the solution for the dual problem of Problem (9) with $k=i$, which corresponds to the $j$ th IT constraint, and from the objective function of Problem (9),

$$
\frac{\partial C_{i}\left(\boldsymbol{\Gamma}_{i}\right)}{\partial \Gamma_{j i}}=\frac{-\boldsymbol{h}_{i i}^{H} \boldsymbol{S}_{i}^{\star} \boldsymbol{h}_{i i}}{\ln 2\left(\sum_{l \neq i} \Gamma_{l i}+\sigma_{i}^{2}\right)\left(\sum_{l \neq i} \Gamma_{l i}+\sigma_{i}^{2}+\boldsymbol{h}_{i i}^{H} \boldsymbol{S}_{i}^{\star} \boldsymbol{h}_{i i}\right)}
$$

where $\boldsymbol{S}_{i}^{\star}$ is the optimal solution of Problem (9) with $k=i$. Similarly, $\frac{\partial C_{j}\left(\boldsymbol{\Gamma}_{j}\right)}{\partial \Gamma_{i j}}$ and $\frac{\partial C_{j}\left(\boldsymbol{\Gamma}_{j}\right)}{\partial \Gamma_{j i}}$ can be obtained from solving Problem (9) via the Lagrange duality method with $k=j$.

From Proposition 4.1, the following observations can be easily obtained (the proofs are omitted for brevity):

- For any particular $\Gamma$ that corresponds to a Pareto-optimal rate-tuple, it must hold that $\Gamma_{i j} \leq \bar{\Gamma}_{i j}, \forall i, j, i \neq j$, where $\bar{\Gamma}_{i j}=\frac{\left\|\boldsymbol{h}_{i j}^{H} \boldsymbol{h}_{i i}\right\|^{2} P_{i}}{\left\|\boldsymbol{h}_{i i}\right\|^{2}}$ corresponds to the case of using maximum transmit power with MRT beamforming for the $i$ th BS;

- For any particular $\boldsymbol{\Gamma}$ that corresponds to a Pareto-optimal rate-tuple, it must hold that $\boldsymbol{h}_{i j}^{H} \boldsymbol{S}_{i}^{\star} \boldsymbol{h}_{i j}=\Gamma_{i j}, \forall i, j, i \neq j$, i.e., the IT constraints across all BSs must be tight.

From the above observations, we see that if we are only interested in the values of $\boldsymbol{\Gamma}$ that correspond to Paretooptimal rate-tuples for the MISO-IC, it is sufficient for us to focus on the subset of $\boldsymbol{\Gamma}$ within the set $\boldsymbol{\Gamma} \geq 0$, in which $\Gamma_{i j} \leq \bar{\Gamma}_{i j}$ and $\Gamma_{i j}=\boldsymbol{h}_{i j}^{H} \boldsymbol{S}_{i}^{\star} \boldsymbol{h}_{i j}, \forall i, j, i \neq j$.

Based on Proposition 4.1, we can develop a simple rule for different pairs of BSs in the cooperative multi-cell system to update their mutual IT constraints for improving both of their transmit rates, while keeping those of the other BSs unchanged. From the proof of Proposition 4.1 given in Appendix $\mathrm{C}$, it follows that the method for any updating BS pair $(i, j)$ to fulfill the above requirements is via changing $\Gamma_{i j}$ and $\Gamma_{j i}$ according to (38). Note that in 


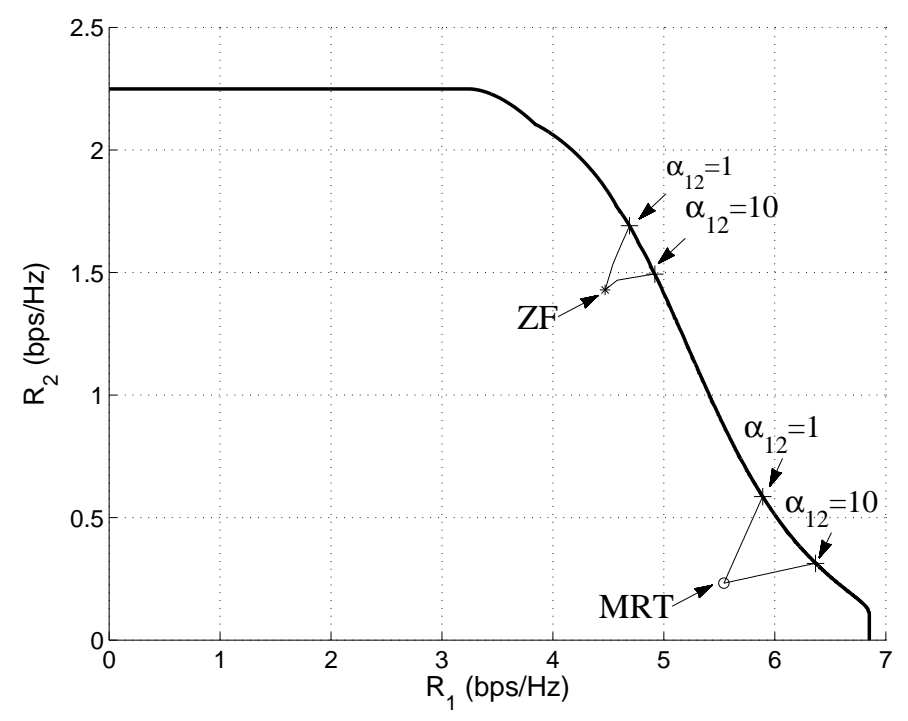

Fig. 2. Achievable rates for the proposed algorithm in a two-user MISO Gaussian IC with interference treated as noise.

general, the choice for $\boldsymbol{d}_{i j}$ in (38) to make $\boldsymbol{D}_{i j} \boldsymbol{d}_{i j}>0$ is not unique. For notational conciseness, let $\boldsymbol{D}_{i j}=\left[\begin{array}{cc}a & b \\ c & d\end{array}\right]$; it can then be shown that one particular choice for $\boldsymbol{d}_{i j}$ is

$$
\boldsymbol{d}_{i j}=\operatorname{sign}(a d-b c) \cdot\left[\alpha_{i j} d-b, a-\alpha_{i j} c\right]^{T}
$$

where $\operatorname{sign}(x)=1$ if $x \geq 0$ and $=-1$ otherwise; $\alpha_{i j} \geq 0$ is a constant that controls the ratio between the rate increments for the $i$ th and $j$ th BSs. It can be easily verified that when $\alpha_{i j}>1$, a larger rate increment is resulted for the $i$ th BS than that for the $j$ th BS, and vice versa when $\alpha_{i j}<1$ (provided that the step-size $\delta_{i j}$ in $(38)$ is sufficiently small).

More specifically, the procedure for any BS pair $(i, j), i \neq j, i \in\{1, \ldots, K\}$, and $j \in\{1, \ldots, K\}$, to update their mutual IT constraints is given as follows. First, the $i$ th BS computes the elements $a$ and $b$ in $\boldsymbol{D}_{i j}$ according to (14) and (15), respectively, with the present value of $\boldsymbol{\Gamma}_{i}$. Similarly, the $j$ th BS computes $c$ and $d$ with the present value of $\boldsymbol{\Gamma}_{j}$. Next, the $i$ th BS sends $a$ and $b$ to the $j$ th BS, while the $j$ th BS sends $c$ and $d$ to the $i$ th BS. Then, assuming that $\alpha_{i j}$ and $\delta_{i j}$ are preassigned values known to these two BSs, they can both compute $\boldsymbol{d}_{i j}$ according to (16) and update $\Gamma_{i j}$ and $\Gamma_{j i}$ according to (38) in Appendix C] Last, with the updated values $\Gamma_{i j}^{\prime}$ and $\Gamma_{j i}^{\prime}$, these two BSs reset their respective beamforming vectors and transmit rates via solving (9) independently. Note that the above operation requires only local information (scalar) exchanges between different pairs of BSs, and thus can be implemented at a very low cost in a cellular system. One version of the decentralized algorithm for cooperative downlink beamforming in a multi-cell system is described in Table I Since in each iteration of the algorithm the achievable rates for the pair of updating BSs both improve and those for all other BSs are unaffected (non-decreasing), and the maximum achievable rates for all BSs are bounded by finite Pareto-optimal values, the convergence of this algorithm is ensured. 


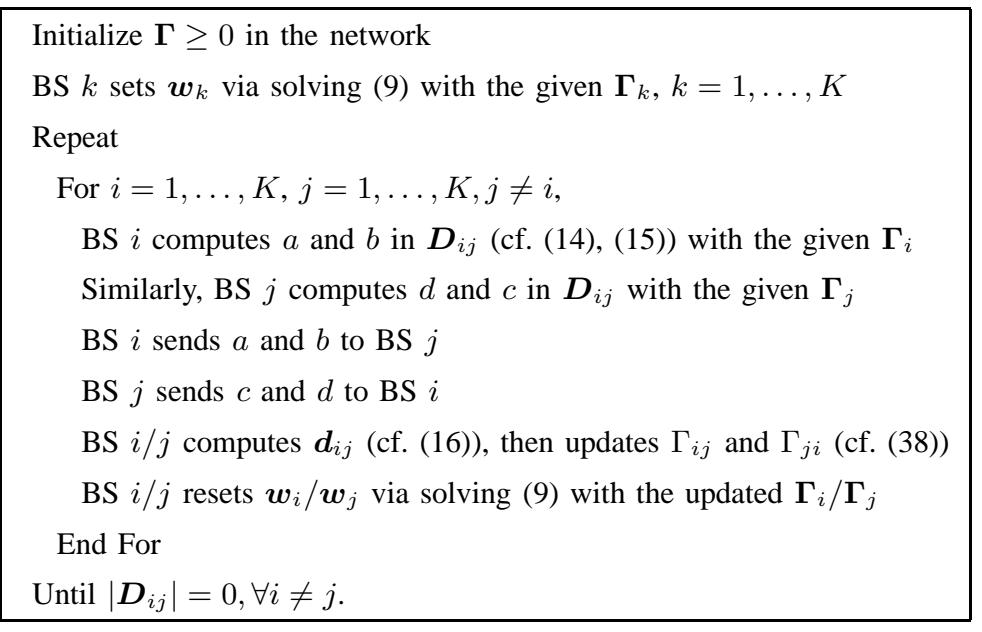

TABLE I

Algorithm For CoOperative Downlink BeAmForming.

Example 4.1: In Fig. 2 (with the same two-user MISO-IC as for Fig. 11), we show the Pareto boundary for an example MISO-IC with $K=2, M_{1}=M_{2}=3, P_{1}=5, P_{2}=1$, and $\sigma_{1}^{2}=\sigma_{2}^{2}=1$, which is obtained by the proposed method in this correspondence, i.e., solving the problems given in (9) for $k=1,2$, and a given pair of values $\Gamma_{12}$ and $\Gamma_{21}$ with $0 \leq \Gamma_{12} \leq \bar{\Gamma}_{12}$ and $0 \leq \Gamma_{21} \leq \bar{\Gamma}_{21}$, and then taking a closure operation over the resultant rate-pairs with all different values of $\Gamma_{12}$ and $\Gamma_{21}$ within their respective ranges. We demonstrate the effectiveness of the proposed decentralized algorithm for implementing the multi-cell cooperative downlink beamforming with two initial rate-pairs, indicated by "ZF" and "MRT" in Fig. 2, which are obtained when both BSs adopt the ZF and the MRT beamforming vectors, respectively, with their maximum transmit powers. It is observed that the achievable rates for both MSs increase with iterations and finally converge to a Pareto-optimal rate-pair 4 By comparing the two cases with $\alpha_{12}=1$ and $\alpha_{12}=10$, it is observed that a larger value of $\alpha_{12}$ results in larger rate values for the first MS in the converged rate-pairs, which is in accordance with our previous discussion.

\section{CONCluding Remarks}

In this correspondence, based on the concept of interference temperature (IT) and under a cellular downlink setup, we have developed a new method to characterize the complete Pareto boundary of the achievable rate region for the $K$-user Gaussian MISO-IC with interference treated as noise. It is shown that the proposed method also leads to a new decentralized algorithm for implementing the downlink beamforming in a cooperative multi-cell system to achieve maximal rates with a prescribed fairness guarantee.

\footnotetext{
${ }^{4}$ We have verified with a large number of random channels and a variety of system parameters that the proposed algorithm always converges to Pareto-optimal rate-pairs for the two-user MISO-IC with randomly selected initial rate-pairs. However, we could not prove this result in general by, e.g., showing that the conditions given in Proposition 4.1 are not only necessary (as proved in this work) but also sufficient for any given $\Gamma$ to achieve a Pareto-optimal rate-tuple for the MISO-IC.
} 
There are a number of directions along which the developed results in this work can be further investigated. First, it would be interesting to extend the multi-cell cooperative beamforming design based on the principle of IT to the scenario where each BS supports simultaneous transmissions to multiple active MSs each with a single antenna or multiple antennas. Second, it remains yet to be proved whether the necessary conditions derived in this work for any particular set of IT constraints across the BSs to guarantee a Pareto-optimal rate-tuple for the MISO-IC are also sufficient, even for the special two-user case. This proof is essential for the proposed downlink beamforming algorithm to achieve the global convergence (Pareto-optimal rates). Last but not least, it is pertinent to analyze the proposed decentralized algorithm that iteratively updates the mutual IT constraints between different pairs of BSs from a game-theoretical viewpoint.

\section{APPENDIX A}

\section{ProOF OF PROPOSITION 3.1}

It can be verified that Problem (9) is convex, and thus it can be solved by the standard Lagrange duality method [16]. Let $\lambda_{k j}, j \neq k$, and $\lambda_{k k}$ be the non-negative dual variables for Problem (9) associated with the $k$ th BS's IT constraint for the $j$ th MS and its own transmit-power constraint, respectively. The Lagrangian function for this problem can be written as

$$
L\left(\boldsymbol{S}_{k}, \boldsymbol{\lambda}_{k}\right)=\log \left(1+\frac{\boldsymbol{h}_{k k}^{H} \boldsymbol{S}_{k} \boldsymbol{h}_{k k}}{\sum_{j \neq k} \Gamma_{j k}+\sigma_{k}^{2}}\right)-\sum_{j \neq k} \lambda_{k j}\left(\boldsymbol{h}_{k j}^{H} \boldsymbol{S}_{k} \boldsymbol{h}_{k j}-\Gamma_{k j}\right)-\lambda_{k k}\left(\operatorname{Tr}\left(\boldsymbol{S}_{k}\right)-P_{k}\right)
$$

where $\boldsymbol{\lambda}_{k}=\left[\lambda_{k 1}, \ldots, \lambda_{k K}\right]$. The dual function of Problem (9) is given by

$$
g\left(\boldsymbol{\lambda}_{k}\right)=\max _{\boldsymbol{S}_{k} \succeq 0} L\left(\boldsymbol{S}_{k}, \boldsymbol{\lambda}_{k}\right)
$$

Accordingly, the dual problem is defined as

$$
\min _{\boldsymbol{\lambda}_{\boldsymbol{k}} \geq 0} g\left(\boldsymbol{\lambda}_{k}\right)
$$

where $\lambda_{k} \geq 0$ means component-wise non-negative. Since Problem (9] is convex with strictly feasible points [16], the duality gap between its optimal value and that of the dual problem is zero; thus, Problem (9) can be equivalently solved via solving its dual problem. In order to solve the dual problem, we need to obtain the dual function $g\left(\boldsymbol{\lambda}_{k}\right)$ for any given $\boldsymbol{\lambda}_{k} \geq 0$. This can be done by solving the maximization problem given in (18), which, according to (17), can be explicitly written as (by discarding irrelevant constant terms):

$$
\begin{aligned}
\underset{\boldsymbol{S}_{k} .}{\operatorname{Max}} & \log \left(1+\frac{\boldsymbol{h}_{k k}^{H} \boldsymbol{S}_{k} \boldsymbol{h}_{k k}}{\sum_{j \neq k} \Gamma_{j k}+\sigma_{k}^{2}}\right)-\operatorname{Tr}\left(\boldsymbol{B}_{k}\left(\boldsymbol{\lambda}_{k}\right) \boldsymbol{S}_{k}\right) \\
\text { s.t. } & \boldsymbol{S}_{k} \succeq 0
\end{aligned}
$$


where $\boldsymbol{B}_{k}\left(\boldsymbol{\lambda}_{k}\right) \triangleq \sum_{j \neq k} \lambda_{k j} \boldsymbol{h}_{k j} \boldsymbol{h}_{k j}^{H}+\lambda_{k k} \boldsymbol{I}$ and $\boldsymbol{B}_{k}\left(\boldsymbol{\lambda}_{k}\right) \succeq 0$ of dimension $M_{k} \times M_{k}$. In order for Problem (20) to have a bounded objective value, it is shown as follows that $\boldsymbol{B}_{k}\left(\boldsymbol{\lambda}_{k}\right)$ should be a full-rank matrix. Suppose that $\boldsymbol{B}_{k}\left(\boldsymbol{\lambda}_{k}\right)$ is rank-deficient, such that we could define $\boldsymbol{S}_{k}=q_{k} \boldsymbol{v}_{k} \boldsymbol{v}_{k}^{H}$, where $q_{k} \geq 0$ and $\boldsymbol{v}_{k} \in \mathbb{C}^{M_{k} \times 1}$ satisfying $\left\|\boldsymbol{v}_{k}\right\|=1$ and $\boldsymbol{B}_{k}\left(\boldsymbol{\lambda}_{k}\right) \boldsymbol{v}_{k}=\mathbf{0}$. Thereby, the objective function of Problem (20) reduces to

$$
\log \left(1+\frac{q_{k}\left\|\boldsymbol{h}_{k k}^{H} \boldsymbol{v}_{k}\right\|^{2}}{\sum_{j \neq k} \Gamma_{j k}+\sigma_{k}^{2}}\right)
$$

Due to the independence of $\boldsymbol{h}_{k k}$ and $\boldsymbol{h}_{k j}$ 's, and thus the independence of $\boldsymbol{h}_{k k}$ and $\boldsymbol{v}_{k}$, it follows that $\left\|\boldsymbol{h}_{k k}^{H} \boldsymbol{v}_{k}\right\|>0$ with probability one such that (21) goes to infinity by letting $q_{k} \rightarrow \infty$. Since the optimal value of Problem (9) must be bounded, without loss of generality, we only need to consider the subset of $\boldsymbol{\lambda}_{k}$ in the set $\boldsymbol{\lambda}_{k} \geq 0$ to make $\boldsymbol{B}_{k}\left(\boldsymbol{\lambda}_{k}\right)$ full-rank.

Remark 2: Note that from the definition of $\boldsymbol{B}_{k}\left(\boldsymbol{\lambda}_{k}\right)$ and the Karush-Kuhn-Tucker (KKT) optimality conditions [16] of Problem (9), it follows that $\boldsymbol{B}_{k}\left(\boldsymbol{\lambda}_{k}\right)$ is full-rank only when either of the following two cases occurs:

- $\lambda_{k k}>0$ : In this case, the transmit power constraint for the $k$ th BS is tight for Problem (9);

- $\lambda_{k k}=0$, but there are at least $M_{k} \lambda_{k j}$ 's, $j \neq k$, having $\lambda_{k j}>0$ : In this case, regardless of whether the transmit power constraint for the $k$ th BS is tight, there are at least $M_{k}$ out of the $K-1$ IT constraints of the $k$ th BS are tight in Problem (9). Note that this case can be true only when $M_{k} \leq K-1$.

From the above discussions, it is known that $\left(\boldsymbol{B}_{k}\left(\boldsymbol{\lambda}_{k}\right)\right)^{-1}$ exists. Thus, we can introduce a new variable $\overline{\boldsymbol{S}}_{k}$ for Problem (20) as

$$
\overline{\boldsymbol{S}}_{k}=\left(\boldsymbol{B}_{k}\left(\boldsymbol{\lambda}_{k}\right)\right)^{1 / 2} \boldsymbol{S}_{k}\left(\boldsymbol{B}_{k}\left(\boldsymbol{\lambda}_{k}\right)\right)^{1 / 2}
$$

and substituting it into (20) yields

$$
\begin{aligned}
\text { Max. } & \log \left(1+\frac{\boldsymbol{h}_{k k}^{H}\left(\boldsymbol{B}_{k}\left(\boldsymbol{\lambda}_{k}\right)\right)^{-1 / 2} \overline{\boldsymbol{S}}_{k}\left(\boldsymbol{B}_{k}\left(\boldsymbol{\lambda}_{k}\right)\right)^{-1 / 2} \boldsymbol{h}_{k k}}{\sum_{j \neq k} \Gamma_{j k}+\sigma_{k}^{2}}\right)-\operatorname{Tr}\left(\overline{\boldsymbol{S}}_{k}\right) \\
\text { s.t. } & \overline{\boldsymbol{S}}_{k} \succeq 0 .
\end{aligned}
$$

Without loss of generality, we can express $\overline{\boldsymbol{S}}_{k}$ into its eigenvalue decomposition (EVD) as $\overline{\boldsymbol{S}}_{k}=\boldsymbol{U}_{k} \boldsymbol{\Theta}_{k} \boldsymbol{U}_{k}^{H}$, where $\boldsymbol{U}_{k}=\left[\boldsymbol{u}_{k 1}, \ldots, \boldsymbol{u}_{k M_{k}}\right] \in \mathbb{C}^{M_{k} \times M_{k}}$ is unitary and $\boldsymbol{\Theta}_{k}=\operatorname{Diag}\left(\left[\theta_{k 1}, \ldots, \theta_{k M_{k}}\right]\right) \succeq 0$. Substituting the ED of $\overline{\boldsymbol{S}}_{k}$ into (23) yields

$$
\begin{aligned}
\underset{\boldsymbol{U}_{k}, \dot{\boldsymbol{\Theta}}_{k}}{\operatorname{Max}} & \log \left(1+\frac{\sum_{i=1}^{M_{k}} \theta_{k i}\left\|\boldsymbol{h}_{k k}^{H}\left(\boldsymbol{B}_{k}\left(\boldsymbol{\lambda}_{k}\right)\right)^{-1 / 2} \boldsymbol{u}_{k i}\right\|^{2}}{\sum_{j \neq k} \Gamma_{j k}+\sigma_{k}^{2}}\right)-\sum_{i=1}^{M_{k}} \theta_{k i} \\
\text { s.t. } & \left\|\boldsymbol{u}_{k i}\right\|=1, \forall i, \quad \boldsymbol{u}_{k i}^{H} \boldsymbol{u}_{k l}=0, \forall l \neq i \\
& \theta_{k i} \geq 0, \forall i .
\end{aligned}
$$


For any given $\boldsymbol{U}_{k}$, it can be verified that the optimal solution of $\boldsymbol{\Theta}_{k}$ for Problem (24) is given by

$$
\theta_{k i}= \begin{cases}\left(\frac{1}{\ln 2}-\frac{\sum_{j \neq k} \Gamma_{j k}+\sigma_{k}^{2}}{\left\|\boldsymbol{h}_{k k}^{H}\left(\boldsymbol{B}_{k}\left(\boldsymbol{\lambda}_{k}\right)\right)^{-1 / 2} \boldsymbol{u}_{k i}\right\|^{2}}\right)^{+} & \text {if } i=\arg \max _{l \in\left\{1, \ldots, M_{k}\right\}}\left\|\boldsymbol{h}_{k k}^{H}\left(\boldsymbol{B}_{k}\left(\boldsymbol{\lambda}_{k}\right)\right)^{-1 / 2} \boldsymbol{u}_{k l}\right\| \\ 0 & \text { otherwise. }\end{cases}
$$

Thus, it follows that for the optimal solution of Problem (23), $\operatorname{Rank}\left(\overline{\boldsymbol{S}}_{k}\right) \leq 1$. Furthermore, let $i^{\prime}$ denote the index of $i$ for which $\theta_{k i^{\prime}} \geq 0$. The objective function of Problem (24) reduces to

$$
\log \left(1+\frac{\theta_{k i^{\prime}}\left\|\boldsymbol{h}_{k k}^{H}\left(\boldsymbol{B}_{k}\left(\boldsymbol{\lambda}_{k}\right)\right)^{-1 / 2} \boldsymbol{u}_{k i^{\prime}}\right\|^{2}}{\sum_{j \neq k} \Gamma_{j k}+\sigma_{k}^{2}}\right)-\theta_{k i^{\prime}}
$$

Clearly, the above function is maximized with any $\theta_{k i^{\prime}}>0$ when

$$
\boldsymbol{u}_{k i^{\prime}}=\frac{\left(\boldsymbol{B}_{k}\left(\boldsymbol{\lambda}_{k}\right)\right)^{-1 / 2} \boldsymbol{h}_{k k}}{\left\|\left(\boldsymbol{B}_{k}\left(\boldsymbol{\lambda}_{k}\right)\right)^{-1 / 2} \boldsymbol{h}_{k k}\right\|}
$$

From (25) and (27), it follows that the optimal solution for Problem (23) is

$$
\overline{\boldsymbol{S}}_{k}=\frac{\left(\frac{1}{\ln 2}-\frac{\sum_{j \neq k} \Gamma_{j k}+\sigma_{k}^{2}}{\left\|\boldsymbol{h}_{k k}^{H}\left(\boldsymbol{B}_{k}\left(\boldsymbol{\lambda}_{k}\right)\right)^{-1 / 2}\right\|^{2}}\right)^{+}}{\left\|\left(\boldsymbol{B}_{k}\left(\boldsymbol{\lambda}_{k}\right)\right)^{-1 / 2} \boldsymbol{h}_{k k}\right\|^{2}}\left(\boldsymbol{B}_{k}\left(\boldsymbol{\lambda}_{k}\right)\right)^{-1 / 2} \boldsymbol{h}_{k k} \boldsymbol{h}_{k k}^{H}\left(\boldsymbol{B}_{k}\left(\boldsymbol{\lambda}_{k}\right)\right)^{-1 / 2} .
$$

Combining the above solution and (22), it can be easily shown that the optimal solution $\boldsymbol{S}_{k}$ for Problem (9) is as given by Proposition 3.1 .

With the obtained dual function $g\left(\boldsymbol{\lambda}_{k}\right)$ for any given $\boldsymbol{\lambda}_{\boldsymbol{k}}$, the dual problem (19) can be solved by searching over $\boldsymbol{\lambda}_{\boldsymbol{k}} \geq 0$ to minimize $g\left(\boldsymbol{\lambda}_{k}\right)$. This can be done via, e.g., the ellipsoid method [22], by utilizing the subgradient of $g\left(\boldsymbol{\lambda}_{k}\right)$ that is obtained from (17) as $\Gamma_{k j}-\boldsymbol{h}_{k j}^{H} \boldsymbol{S}_{k}^{*} \boldsymbol{h}_{k j}$ for $\lambda_{k j}, j \neq k$ and $P_{k}-\operatorname{Tr}\left(\boldsymbol{S}_{k}^{*}\right)$ for $\lambda_{k k}$, where $\boldsymbol{S}_{k}^{*}$ is the optimal solution for Problem (20) with the given $\boldsymbol{\lambda}_{k}$. When $\boldsymbol{\lambda}_{\boldsymbol{k}}$ converges to the optimal solution for the dual problem, the corresponding $S_{k}^{*}$ becomes the optimal solution for Problem (9). Proposition 3.1 thus follows.

\section{APPENDIX B}

\section{Proof of Proposition 3.2}

Since the given set of $\boldsymbol{S}_{1}, \ldots, \boldsymbol{S}_{K}$ achieves the Pareto-optimal rate-tuple $\left(R_{1}, \ldots, R_{K}\right)$ for the MISO-IC, from (2) and (3) it follows that for any $k \in\{1, \ldots, K\}$

$$
R_{k}=\log \left(1+\frac{\boldsymbol{h}_{k k}^{H} \boldsymbol{S}_{k} \boldsymbol{h}_{k k}}{\sum_{j \neq k} \boldsymbol{h}_{j k}^{H} \boldsymbol{S}_{j} \boldsymbol{h}_{j k}+\sigma_{k}^{2}}\right) .
$$

Since $\Gamma_{j k}=\boldsymbol{h}_{j k}^{H} \boldsymbol{S}_{j} \boldsymbol{h}_{j k}, \forall j \neq k$, (29) can be rewritten as

$$
R_{k}=\log \left(1+\frac{\boldsymbol{h}_{k k}^{H} \boldsymbol{S}_{k} \boldsymbol{h}_{k k}}{\sum_{j \neq k} \Gamma_{j k}+\sigma_{k}^{2}}\right) .
$$

Note that (30) is the same as the objective function of Problem (9) for the given $k$. Furthermore, from (3) it follows that $\operatorname{Tr}\left(\boldsymbol{S}_{k}\right) \leq P_{k}$. Using this and the fact that $\Gamma_{k j}=\boldsymbol{h}_{k j}^{H} \boldsymbol{S}_{k} \boldsymbol{h}_{k j}, \forall j \neq k$, it follows that $\boldsymbol{S}_{k}$ satisfies the constraints 
given in Problem (9) for the given $k$. Therefore, $\boldsymbol{S}_{k}$ must be a feasible solution for Problem (9) with the given $k$ and $\Gamma_{k}$.

Next, we need to prove that $\boldsymbol{S}_{k}$ is indeed the optimal solution of Problem (9) for any given $k$, and thus the corresponding achievable rate $R_{k}$ is equal to the optimal value of Problem (9), which is $C_{k}\left(\boldsymbol{\Gamma}_{k}\right)$. We prove this result by contradiction. Suppose that the optimal solution for Problem (9), denoted by $\boldsymbol{S}_{k}^{\star}$, is not equal to $\boldsymbol{S}_{k}$ for a given $k$.

Thus, we have

$$
\begin{aligned}
R_{k} & <\log \left(1+\frac{\boldsymbol{h}_{k k}^{H} \boldsymbol{S}_{k}^{\star} \boldsymbol{h}_{k k}}{\sum_{j \neq k} \Gamma_{j k}+\sigma_{k}^{2}}\right) \\
& =\log \left(1+\frac{\boldsymbol{h}_{k k}^{H} \boldsymbol{S}_{k}^{\star} \boldsymbol{h}_{k k}}{\sum_{j \neq k} \boldsymbol{h}_{j k}^{H} \boldsymbol{S}_{j} \boldsymbol{h}_{j k}+\sigma_{k}^{2}}\right) \triangleq r_{k} .
\end{aligned}
$$

Furthermore, since $\boldsymbol{h}_{k j}^{H} \boldsymbol{S}_{k}^{\star} \boldsymbol{h}_{k j} \leq \Gamma_{k j}, \forall j \neq k$, we have for any $j \neq k$,

$$
\begin{aligned}
R_{j} & =\log \left(1+\frac{\boldsymbol{h}_{j j}^{H} \boldsymbol{S}_{j} \boldsymbol{h}_{j j}}{\sum_{i \neq j} \boldsymbol{h}_{i j}^{H} \boldsymbol{S}_{i} \boldsymbol{h}_{i j}+\sigma_{j}^{2}}\right) \\
& =\log \left(1+\frac{\boldsymbol{h}_{j j}^{H} \boldsymbol{S}_{j} \boldsymbol{h}_{j j}}{\sum_{i \neq j} \Gamma_{i j}+\sigma_{j}^{2}}\right) \\
& \leq \log \left(1+\frac{\boldsymbol{h}_{j j}^{H} \boldsymbol{S}_{j} \boldsymbol{h}_{j j}}{\sum_{i \neq j, k} \Gamma_{i j}+\boldsymbol{h}_{k j}^{H} \boldsymbol{S}_{k}^{\star} \boldsymbol{h}_{k j}+\sigma_{j}^{2}}\right) \\
& =\log \left(1+\frac{\boldsymbol{h}_{j j}^{H} \boldsymbol{S}_{j} \boldsymbol{h}_{j j}}{\sum_{i \neq j, k} \boldsymbol{h}_{i j}^{H} \boldsymbol{S}_{i} \boldsymbol{h}_{i j}+\boldsymbol{h}_{k j}^{H} \boldsymbol{S}_{k}^{\star} \boldsymbol{h}_{k j}+\sigma_{j}^{2}}\right) \triangleq r_{j} .
\end{aligned}
$$

Thus, for another set of transmit covariance matrices given by $\boldsymbol{S}_{1}, \ldots, \boldsymbol{S}_{k-1}, \boldsymbol{S}_{k}^{\star}, \boldsymbol{S}_{k+1}, \ldots, \boldsymbol{S}_{K}$, the corresponding achievable rate-tuple for the MISO-IC, $\left(r_{1}, \ldots, r_{K}\right)$, satisfies that $r_{k}>R_{k}$ and $r_{j} \geq R_{j}, \forall j \neq k$, which contradicts the fact that $\left(R_{1}, \ldots, R_{K}\right)$ is a Pareto-optimal rate-tuple for the MISO-IC. Hence, the presumption that $\boldsymbol{S}_{k} \neq \boldsymbol{S}_{k}^{\star}$ for any given $k$ cannot be true. Thus, we have $\boldsymbol{S}_{k}=\boldsymbol{S}_{k}^{\star}$ and $R_{k}=C_{k}\left(\boldsymbol{\Gamma}_{k}\right), \forall k$. Proposition 3.2 thus follows.

\section{APPENDIX C}

\section{PROOF OF PROPOSITION 4.1}

As given in Proposition 4.1, with $\boldsymbol{\Gamma}$, the corresponding optimal values of the problems in (9) for all $k$ 's, $C_{k}\left(\boldsymbol{\Gamma}_{k}\right)$ 's, correspond to a Pareto-optimal rate-tuple for the MISO-IC, denoted by $\left(R_{1}, \ldots, R_{K}\right)$. Let $S_{1}, \ldots, S_{K}$ denote the set of optimal solutions for the problems in (9). We thus have

$$
C_{k}\left(\boldsymbol{\Gamma}_{k}\right)=R_{k}=\log \left(1+\frac{\boldsymbol{h}_{k k}^{H} \boldsymbol{S}_{k} \boldsymbol{h}_{k k}}{\sum_{j \neq k} \Gamma_{j k}+\sigma_{k}^{2}}\right), k=1, \ldots, K .
$$

Next, we prove Proposition 4.1 by contradiction. Suppose that there exists a pair of $(i, j)$ with $\left|\boldsymbol{D}_{i j}\right| \neq 0$, where $\boldsymbol{D}_{i j}$

is defined in (13). Define a new $\boldsymbol{\Gamma}^{\prime}$ over $\boldsymbol{\Gamma}$, where all the elements in $\boldsymbol{\Gamma}$ remain unchanged except $\left[\Gamma_{i j}, \Gamma_{j i}\right]^{T}$ being replaced by

$$
\left[\Gamma_{i j}^{\prime}, \Gamma_{j i}^{\prime}\right]^{T}=\left[\Gamma_{i j}, \Gamma_{j i}\right]^{T}+\delta_{i j} \cdot \boldsymbol{d}_{i j}
$$


where $\delta_{i j}>0$ is a small step-size, and $\boldsymbol{d}_{i j}$ is any vector that satisfies $\boldsymbol{D}_{i j} \boldsymbol{d}_{i j}>0$ (component-wise), with one possible value for such $\boldsymbol{d}_{i j}$ is given by (16) in the main text. With $\boldsymbol{\Gamma}^{\prime}$, the optimal solutions for the problems in (9) remain unchanged $\forall k \neq i, j$, while for those with $k=i$ and $k=j$, the optimal solutions are changed to be $\boldsymbol{S}_{i}^{\star}$ and $\boldsymbol{S}_{j}^{\star}$, respectively. Accordingly, the new achievable rates in the MISO-IC for any $k \neq i, j$ are given by

$$
\begin{aligned}
r_{k} & =\log \left(1+\frac{\boldsymbol{h}_{k k}^{H} \boldsymbol{S}_{k} \boldsymbol{h}_{k k}}{\sum_{l \neq k, i, j} \boldsymbol{h}_{l k}^{H} \boldsymbol{S}_{l} \boldsymbol{h}_{l k}+\boldsymbol{h}_{i k}^{H} \boldsymbol{S}_{i}^{\star} \boldsymbol{h}_{i k}+\boldsymbol{h}_{j k}^{H} \boldsymbol{S}_{j}^{\star} \boldsymbol{h}_{j k}+\sigma_{k}^{2}}\right) \\
& =\log \left(1+\frac{\boldsymbol{h}_{k k}^{H} \boldsymbol{S}_{k} \boldsymbol{h}_{k k}}{\sum_{l \neq k, i, j} \Gamma_{l k}+\boldsymbol{h}_{i k}^{H} \boldsymbol{S}_{i}^{\star} \boldsymbol{h}_{i k}+\boldsymbol{h}_{j k}^{H} \boldsymbol{S}_{j}^{\star} \boldsymbol{h}_{j k}+\sigma_{k}^{2}}\right) \\
& \geq R_{k}
\end{aligned}
$$

where (41) is due to (37) and the facts that $\boldsymbol{h}_{i k}^{H} \boldsymbol{S}_{i}^{\star} \boldsymbol{h}_{i k} \leq \Gamma_{i k}$ and $\boldsymbol{h}_{j k}^{H} \boldsymbol{S}_{j}^{\star} \boldsymbol{h}_{j k} \leq \Gamma_{j k}$. Also, it can be shown that

$$
\begin{aligned}
r_{i} & =\log \left(1+\frac{\boldsymbol{h}_{i i}^{H} \boldsymbol{S}_{i}^{\star} \boldsymbol{h}_{i i}}{\sum_{l \neq i, j} \boldsymbol{h}_{l i}^{H} \boldsymbol{S}_{l} \boldsymbol{h}_{l i}+\boldsymbol{h}_{j i}^{H} \boldsymbol{S}_{j}^{\star} \boldsymbol{h}_{j i}+\sigma_{i}^{2}}\right) \\
& =\log \left(1+\frac{\boldsymbol{h}_{i i}^{H} \boldsymbol{S}_{i}^{\star} \boldsymbol{h}_{i i}}{\sum_{l \neq i, j} \Gamma_{l i}+\boldsymbol{h}_{j i}^{H} \boldsymbol{S}_{j}^{\star} \boldsymbol{h}_{j i}+\sigma_{i}^{2}}\right) \\
& \geq C_{i}\left(\boldsymbol{\Gamma}_{i}^{\prime}\right)
\end{aligned}
$$

where (44) is due to the facts that $\boldsymbol{h}_{j i}^{H} \boldsymbol{S}_{j}^{\star} \boldsymbol{h}_{j i} \leq \Gamma_{j i}^{\prime}$ and $\boldsymbol{S}_{i}^{\star}$ achieves the optimal value of Problem (9) with $k=i$ and the given $\boldsymbol{\Gamma}_{i}^{\prime}$, denoted by $C_{i}\left(\boldsymbol{\Gamma}_{i}^{\prime}\right)$. Similarly, it can be shown that $r_{j} \geq C_{j}\left(\boldsymbol{\Gamma}_{j}^{\prime}\right)$. Thus, from $\underline{38}$ and $\boldsymbol{D}_{i j} \boldsymbol{d}_{i j}>0$, it follows that with sufficiently small $\delta_{i j}$

$$
\begin{aligned}
{\left[\begin{array}{l}
r_{i} \\
r_{j}
\end{array}\right] } & \geq\left[\begin{array}{l}
C_{i}\left(\boldsymbol{\Gamma}_{i}^{\prime}\right) \\
C_{j}\left(\boldsymbol{\Gamma}_{j}^{\prime}\right)
\end{array}\right] \\
& \cong\left[\begin{array}{c}
C_{i}\left(\boldsymbol{\Gamma}_{i}\right) \\
C_{j}\left(\boldsymbol{\Gamma}_{j}\right)
\end{array}\right]+\delta_{i j} \boldsymbol{D}_{i j} \boldsymbol{d}_{i j} \\
& >\left[\begin{array}{c}
R_{i} \\
R_{j}
\end{array}\right] .
\end{aligned}
$$

Therefore, we have found a new set of achievable rate-tuple for the MISO-IC with $\boldsymbol{\Gamma}^{\prime},\left(r_{1}, \ldots, r_{K}\right)$, which has $r_{i}>R_{i}, r_{j}>R_{j}$, and $r_{k} \geq R_{k}, \forall k \neq i, j$. Clearly, this contradicts the fact that $\left(R_{1}, \ldots, R_{K}\right)$ is Pareto-optimal for the MISO-IC. Thus, the presumption that there exists a pair of $(i, j)$ with $\left|\boldsymbol{D}_{i j}\right| \neq 0$ cannot be true. Proposition 4.1 thus follows.

\section{REFERENCES}

[1] S. Shamai (Shitz) and B. M. Zaidel, "Enhancing the cellular downlink capacity via co-processing at the transmitting end," in Proc. IEEE Veh. Technol. Conf. (VTC), vol. 3, pp. 1745-1749, May 2001.

[2] H. Zhang and H. Dai, "Cochannel interference mitigation and cooperative processing in downlink multicell multiuser MIMO networks," EURASIP J. Wireless Commun. Netw., no. 2, pp. 222-235, 2004. 
[3] M. Karakayali, G. J. Foschini, and R. A. Valenzuela, "Network coordination for spectrally efficient communications in cellular systems," IEEE Wireless Commun., vol. 13, no. 4, pp. 56-61, Aug. 2006.

[4] O. Somekh, B. Zaidel, and S. Shamai (Shitz), "Sum rate characterization of joint multiple cell-site processing," IEEE Trans. Inf. Theory, vol. 53, no. 12, pp. 4473-4497, Dec. 2007.

[5] S. Jing, D. Tse, J. Hou, J. Soriaga, J. Smee, and R. Padovani, "Downlink macro-diversity in cellular networks," in Proc. IEEE Int. Symp. Inf. Theory (ISIT), pp. 24-29, June 2007.

[6] R. Zhang, "Cooperative multi-cell block diagonalization with per-base-station power constraints," to appear in IEEE J. Sel. Areas Commun., 2010.

[7] H. Dahrouj and W. Yu, "Coordinated beamforming for the multi-cell multi-antenna wireless system," in Proc. Conf. Inf. Sciences and Systems (CISS), Mar. 2008.

[8] T. S. Han and K. Kobayashi, "A new achievable rate region for the interference channel," IEEE Trans. Inf. Theory, vol. 27, no. 1, pp. 49-60, Jan. 1981.

[9] R. Etkin, D. Tse, and H. Wang, "Gaussian interference channel capacity to within one bit," IEEE Trans. Inf. Theory, vol. 54, no. 12, pp. 5534-5562, Dec. 2008.

[10] E. Jorswieck, E. Larsson, and D. Danev, "Complete characterization of the Pareto boundary for the MISO interference channel," IEEE Trans. Sig. Process., vol. 56, no. 10, pp. 5292-5296, Oct. 2008.

[11] D. Schmidt, C. Shi, R. Berry, M. Honig, and W. Utschick, "Distributed resource allocation schemes: pricing algorithms for power control and beamformer design in interference networks," IEEE Trans. Sig. Process. Mag., vol. 26. no. 5, pp. 53-63, Sep. 2009.

[12] V. R. Cadambe and S. A. Jafar, "Interference alignment and the degrees of freedom for the K user interference channel," IEEE Trans. Inf. Theory, vol. 54, no. 8, pp. 3425-3441, Aug. 2008.

[13] S. Haykin, "Cognitive radio: brain-empowered wireless communications," IEEE J. Sel. Areas Commun., vol. 23, no. 2, pp. 201-220, Feb. 2005.

[14] R. Zhang and Y. C. Liang, "Exploiting multi-antennas for opportunistic spectrum sharing in cognitive radio networks," IEEE J. S. Topics Sig. Process., vol. 2, no. 1, pp. 88-102, Feb. 2008.

[15] X. Shang, B. Chen, and H. Poor "On the optimality of beamforming for multi-user MISO interference channels with single-user detection," in Proc. IEEE Global Commun. Conf. (Globecom), Dec. 2009.

[16] S. Boyd and L. Vandenberghe, Convex optimization, Cambridge University Press, 2004.

[17] V. R. Cadambe, S. A. Jafar, and C. Wang, "Interference alignment with asymmetric complex signaling - settling the Host-Madsen-Nosratinia conjecture," available [online] at arXiv:0904.0274

[18] M. Mohseni, R. Zhang, and J. M. Cioffi, "Optimized transmission for fading multiple-access and broadcast channels with multiple antennas," IEEE J. Sel. Areas Commun., vol. 24, no. 8, pp. 1627-1639, Aug. 2006.

[19] M. Bengtsson and B. Ottersten, "Optimal downlink beamforming using semidefinite optimization", in Proc. Annual Allerton Conf. Commun. Control and Comput., pp. 987-996, Sep. 1999.

[20] M. Grant and S. Boyd, "CVX: Matlab software for disciplined convex programming," available [online] at http://stanford.edu/ boyd/cvx

[21] R. A. Horn and C. R. Johnson, Matrix Analysis, Cambridge University Press, 1985.

[22] R. G. Bland, D. Goldfarb, and M. J. Todd, “The ellipsoid method: a survey,” Operations Research, vol. 29, no. 6, pp. 1039-1091, 1981. 